\title{
MAPPINGS PRESERVING REGULAR HEXAHEDRONS
}

\author{
SOON-MO JUNG AND BYUNGBAE KIM
}

Received 23 May 2005 and in revised form 23 September 2005

We will prove that if a one-to-one mapping $f: \mathbb{R}^{3} \rightarrow \mathbb{R}^{3}$ preserves regular hexahedrons, then $f$ is a linear isometry up to translation.

\section{Introduction}

Let $X$ and $Y$ be normed spaces. A mapping $f: X \rightarrow Y$ is called an isometry if $f$ satisfies the equality

$$
\|f(x)-f(y)\|=\|x-y\|
$$

for all $x, y \in X$. A distance $r>0$ is said to be preserved (conservative) by a mapping $f$ : $X \rightarrow Y$ if $\|f(x)-f(y)\|=r$ for all $x, y \in X$ with $\|x-y\|=r$.

If $f$ is an isometry, then every distance $r>0$ is conservative by $f$, and conversely. We can now raise a question whether each mapping that preserves certain distances is an isometry. Indeed, Aleksandrov [1] had raised a question whether a mapping $f: X \rightarrow X$ preserving a distance $r>0$ is an isometry, which is now known to us as the Aleksandrov problem.

Beckman and Quarles [2] solved the Aleksandrov problem for finite-dimensional real Euclidean spaces $X=\mathbb{R}^{n}$ (see also $[3,4,5,6,7,12,13,14,15,16,17,18,19]$ ).

Theorem 1.1 (Beckman and Quarles). If a mapping $f: \mathbb{R}^{n} \rightarrow \mathbb{R}^{n}(2 \leq n<\infty)$ preserves a distance $r>0$, then $f$ is a linear isometry up to translation.

It seems to be interesting to investigate whether the "distance $r>0$ " in the above theorem can be replaced by some properties characterized by "geometrical figures" without loss of its validity.

In [8], Jung proved that if a one-to-one mapping $f: \mathbb{R}^{n} \rightarrow \mathbb{R}^{n}(n \geq 2)$ maps every regular triangle (quadrilateral or hexagon) of side length $a>0$ onto a figure of the same type with side length $b>0$, then there exists a linear isometry $I: \mathbb{R}^{n} \rightarrow \mathbb{R}^{n}$ up to translation such that $f(x)=(b / a) I(x)$. 


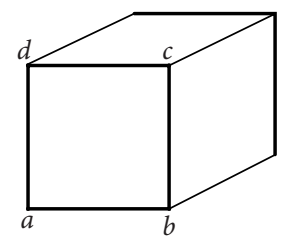

Figure 1.1. Cube A.

Furthermore, the authors [10] proved that if a one-to-one mapping $f: \mathbb{R}^{n} \rightarrow \mathbb{R}^{n}$ maps every unit circle onto a unit circle, then $f$ is a linear isometry up to translation (see also $[9,11])$.

In this connection, we will extend the results of [8] to the more general threedimensional objects, that is, we prove in this paper that if a one-to-one mapping $f: \mathbb{R}^{3} \rightarrow$ $\mathbb{R}^{3}$ maps every regular hexahedron onto a regular hexahedron, then $f$ is a linear isometry up to translation. (An isometry $I: \mathbb{R}^{3} \rightarrow \mathbb{R}^{3}$ is called a linear isometry up to translation if there exists a point $v \in \mathbb{R}^{3}$ such that $I(x)-v$ is a linear mapping.)

\section{Main theorem}

From now on, by a cube we mean a regular hexahedron with side length one. We first make our terms precise as follows. In Figure 1.1, we will call the points $a, b, c, d$ "vertices" and the lines $\overline{a b}, \overline{b c}, \overline{c d}, \overline{d a}$ "edges" and the plane bounded by the four edges $\overline{a b}, \overline{b c}, \overline{c d}$, $\overline{d a}$ "face $a b c d$ " or simply a "face." Further by a cube or hexahedron we will mean the six faces only and not the three-dimensional open set bounded by those six faces. Let us denote the three-dimensional open set bounded by cube $A$ as "Inside of $A$ " or simply as Inside $(A)$.

Suppose that $p \in A$ where $p$ is a point and $A$ is a cube. Firstly let us review the solid angles in three dimensions. If $p$ is a vertex, say $p=a$, then the solid angle that $\operatorname{Inside}(A)$ subtends with respect to $p$ is $\pi / 2$. If $p$ is a point which belongs to an edge and is not a vertex, then the solid angle that $\operatorname{Inside}(A)$ subtends with respect to $p$ is $\pi$. If $p \in A$ is neither a vertex nor an edge point, then the solid angle that Inside $(A)$ subtends with respect to $p$ is $2 \pi$. Let us denote the solid angle that Inside $(A)$ subtends with respect to $p \in A$ by $\Omega(A, p)$. Therefore for $p \in A$, if $\Omega(A, p)=\pi / 2$ or $\Omega(A, p)=\pi, p$ is a vertex of $A$ or $p$ is an edge point of $A$ (and not a vertex), respectively. If $\Omega(A, p)=2 \pi$, then $p$ is neither a vertex nor an edge point of a cube $A$. Now we prove the following lemma.

Lemma 2.1. Let a one-to-one mapping $f: \mathbb{R}^{3} \rightarrow \mathbb{R}^{3}$ map every regular hexahedron onto a regular hexahedron. For any $A$ and $B$ cubes, if $\operatorname{Inside}(A) \cap \operatorname{Inside}(B)=\varnothing$, then Inside $\{f(A)\} \cap \operatorname{Inside}\{f(B)\}=\varnothing$.

Proof. First, we show that if $q \notin \operatorname{Inside}(A)$, then $f(q) \notin \operatorname{Inside}\{f(A)\}$. In other words, we show that if $f(q) \in \operatorname{Inside}\{f(A)\}$, then $q \in \operatorname{Inside}(A)$. Assume that $q \in A$. Then $f(q) \in$ $f(A)$ and so $f(q) \notin \operatorname{Inside}\{f(A)\}$. Suppose that $q \notin \operatorname{Inside}(A)$ and $q \notin A$. Then choose another cube $B$ such that $q \in B$ and $B \cap A=\varnothing$. Then $f(B) \cap f(A)=\varnothing$ and therefore $f(q) \notin \operatorname{Inside}\{f(A)\}$. 


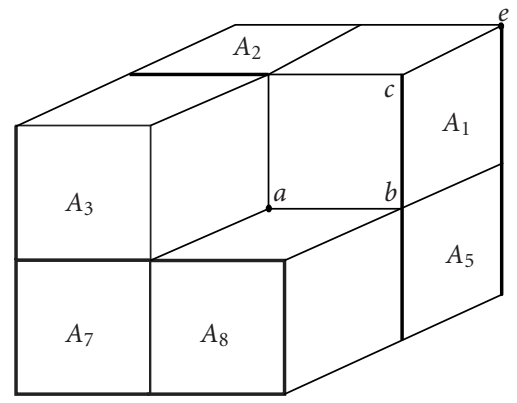

Figure 2.1

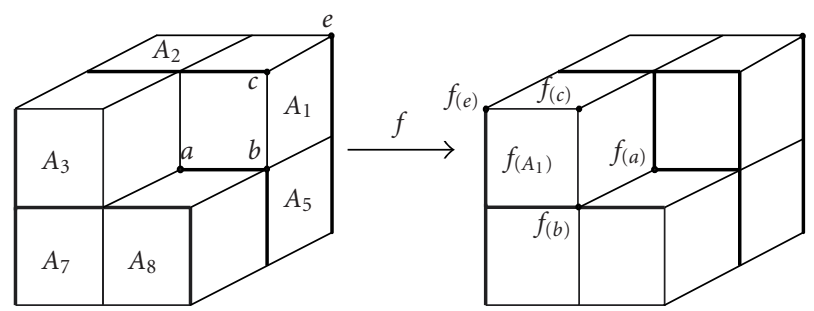

Figure 2.2

Now, let Inside $\{f(A)\} \cap \operatorname{Inside}\{f(B)\} \neq \varnothing$. Then Inside $\{f(A)\} \cap f(B) \neq \varnothing$, which means that for some $b \in B, f(b) \in \operatorname{Inside}\{f(A)\}$. Therefore $b \in \operatorname{Inside}(A)$ and Inside $(A) \cap B \neq \varnothing$ by which we conclude that Inside $(A) \cap \operatorname{Inside}(B) \neq \varnothing$.

We show now that if any one-to-one mapping preserves regular hexahedrons, then it is actually an isometry. More precisely, we have the following.

THeOREM 2.2. If a one-to-one mapping $f: \mathbb{R}^{3} \rightarrow \mathbb{R}^{3}$ maps every regular hexahedron onto a regular hexahedron, then $f$ is a linear isometry up to translation.

Proof. We show that $f$ preserves the distance $\sqrt{3}$. Let $a$ be a vertex of a cube $A=A_{1}$. We can then construct 7 more cubes $A_{i}(i=2, \ldots, 8)$ so that $a$ is the common vertex of 8 cubes $A_{i}(i=1, \ldots, 8)$ and Inside $\left(A_{i}\right) \cap \operatorname{Inside}\left(A_{j}\right)=\varnothing$ for $i \neq j$ (see Figure 2.1). Then $f(a)$ belongs to $f\left(A_{i}\right)$ for $i=1, \ldots, 8$ and by Lemma 2.1 Inside $\left\{f\left(A_{i}\right)\right\} \cap \operatorname{Inside}\left\{f\left(A_{j}\right)\right\}=\varnothing$ for $i \neq j$. Now the solid angle that Inside $\left\{f\left(A_{i}\right)\right\}$ subtends with respect to $f(a)$ is at least $\pi / 2$ for any $i$, that is, $\Omega\left(f\left(A_{i}\right), f(a)\right) \geq \pi / 2$. Since the maximum solid angle with respect to the point $f(a)$ is $4 \pi, \Omega\left(f\left(A_{i}\right), f(a)\right)=\pi / 2$ and $f(a)$ is a vertex of $f\left(A_{i}\right)$ for every $i$. As a conclusion, if $a$ is a vertex of a cube $A$, then $f(a)$ is a vertex of a cube $f(A)$.

Now given any two points $a$ and $e$ which are separated by the distance $\sqrt{3}$ from each other, form cube $A_{1}$ such that they are two vertices of $A_{1}$. We form 7 more cubes $A_{2}, \ldots, A_{8}$ so that the following conditions are met (see Figure 2.2). Firstly, $\operatorname{Inside}\left(A_{i}\right) \cap \operatorname{Inside}\left(A_{j}\right)=$ $\varnothing$ for $i \neq j . a$ is the common vertex of $A_{i}, i=1, \ldots, 8$. Each cube $A_{i}$ has exactly 3 vertices 


\section{Mappings preserving regular hexahedrons}

(like the vertex $b$ ) each of which is the common vertex of exactly four cubes. They are all separated from $a$ by the distance 1 . Each cube $A_{i}$ has exactly 3 vertices (like the vertex $c$ ) each of which is the common vertex of exactly two cubes. They are all separated from $a$ by the distance $\sqrt{2}$. Each cube $A_{i}$ has exactly one vertex (like the vertex $e$ ) which belongs to only one cube $A_{i}$ and is separated from $a$ by the distance $\sqrt{3}$.

If we use Lemma 2.1, we can obtain Inside $\left\{f\left(A_{i}\right)\right\} \cap \operatorname{Inside}\left\{f\left(A_{j}\right)\right\}=\varnothing$ for $i \neq j$. $f(a)$ is the common vertex of $f\left(A_{i}\right), i=1, \ldots, 8$. Each cube $f\left(A_{i}\right)$ has exactly 3 vertices (like the vertex $f(b)$ ) each of which is the common vertex of exactly four cubes. They are all separated from $f(a)$ by the distance 1 . Each cube $f\left(A_{i}\right)$ has exactly 3 vertices (like the vertex $f(c)$ ) each of which is the common vertex of exactly two cubes. They are all separated from $f(a)$ by the distance $\sqrt{2}$. Each cube $f\left(A_{i}\right)$ has exactly one vertex (like the vertex $f(e))$ which belongs to only one cube $f\left(A_{i}\right)$. It is separated from $f(a)$ by the distance $\sqrt{3}$. Therefore, we conclude that the distance between $f(a)$ and $f(e)$ is $\sqrt{3}$.

Consequently, in view of the theorem of Beckman and Quarles, we conclude that $f$ is a linear isometry up to translation.

\section{References}

[1] A. D. Aleksandrov, Mappings of families of sets, Soviet Math. Dokl. 11 (1970), 116-120.

[2] F. S. Beckman and D. A. Quarles Jr., On isometries of Euclidean spaces, Proc. Amer. Math. Soc. 4 (1953), 810-815.

[3] W. Benz, Isometrien in normierten Räumen [Isometries in normed spaces], Aequationes Math. 29 (1985), no. 2-3, 204-209.

[4] An elementary proof of the theorem of Beckman and Quarles, Elem. Math. 42 (1987), no. 1, 4-9.

[5] R. L. Bishop, Characterizing motions by unit distance invariance, Math. Mag. 46 (1973), 148151.

[6] L. Debnath and P. Mikusinski, Introduction to Hilbert Spaces with Applications, 3rd ed., Elsevier Academic Press, California, 2005.

[7] D. Greenwell and P. D. Johnson, Functions that preserve unit distance, Math. Mag. 49 (1976), no. $2,74-79$.

[8] S.-M. Jung, Mappings preserving some geometrical figures, Acta Math. Hungar. 100 (2003), no. 12, 167-175.

[9] - Mappings preserving unit circles in $\mathbb{R}^{2}$, Octogon Math. Mag. 11 (2003), 450-453.

[10] S.-M. Jung and B. Kim, Unit-circle-preserving mappings, Int. J. Math. Math. Sci. 2004 (2004), no. $66,3577-3586$.

[11] Unit-sphere preserving mappings, Glas. Mat. Ser. III 39(59) (2004), no. 2, 327-330.

[12] B. Mielnik and Th. M. Rassias, On the Aleksandrov problem of conservative distances, Proc. Amer. Math. Soc. 116 (1992), no. 4, 1115-1118.

[13] Th. M. Rassias, Unsolved problems: Is a distance one preserving mapping between metric spaces always an isometry?, Amer. Math. Monthly 90 (1983), no. 3, 200.

[14]_- Mappings that preserve unit distance, Indian J. Math. 32 (1990), no. 3, 275-278.

[15] Th. M. Rassias and P. Šemrl, On the Mazur-Ulam theorem and the Aleksandrov problem for unit distance preserving mappings, Proc. Amer. Math. Soc. 118 (1993), no. 3, 919-925.

[16] Th. M. Rassias and C. S. Sharma, Properties of isometries, J. Natur. Geom. 3 (1993), no. 1, 1-38.

[17] E. M. Schröder, Eine Ergänzung zum Satz von Beckman and Quarles, Aequationes Math. 19 (1979), no. 1, 89-92. 
[18] C. G. Townsend, Congruence-preserving mappings, Math. Mag. 43 (1970), 37-38.

[19] S. Xiang, Mappings of conservative distances and the Mazur-Ulam theorem, J. Math. Anal. Appl. 254 (2001), no. 1, 262-274.

Soon-Mo Jung: Mathematics Section, College of Science and Technology, Hong-Ik University, 339-701 Chochiwon, Korea

E-mail address: smjung@wow.hongik.ac.kr

Byungbae Kim: Mathematics Section, College of Science and Technology, Hong-Ik University, 339-701 Chochiwon, Korea

E-mail address: bkim@wow.hongik.ac.kr 


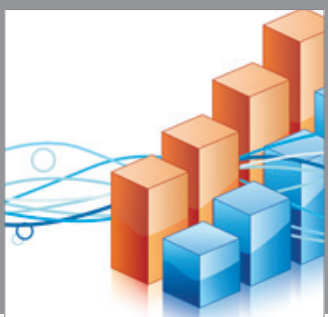

Advances in

Operations Research

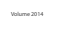

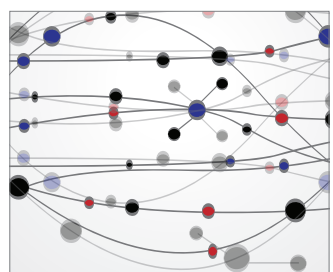

\section{The Scientific} World Journal
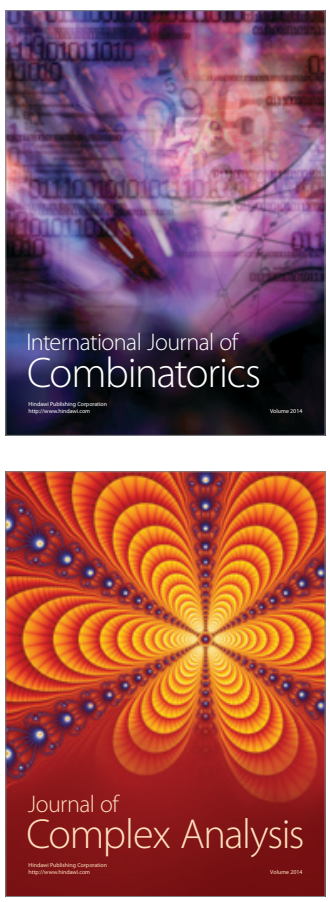

International Journal of

Mathematics and

Mathematical

Sciences
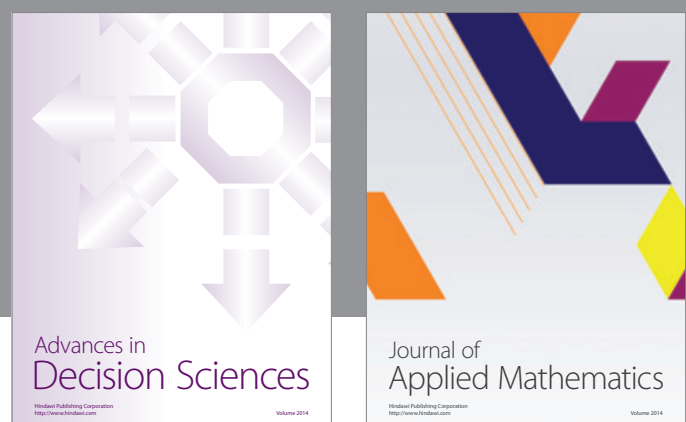

Journal of

Applied Mathematics
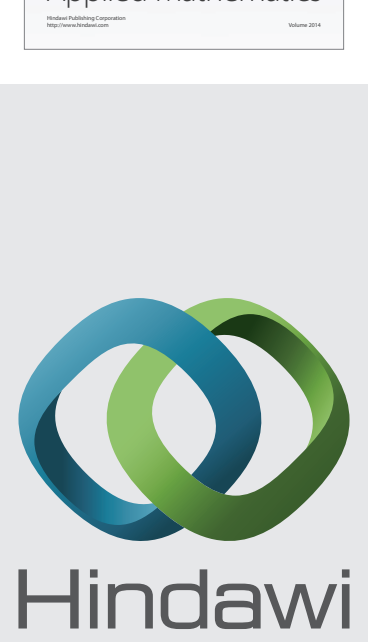

Submit your manuscripts at http://www.hindawi.com
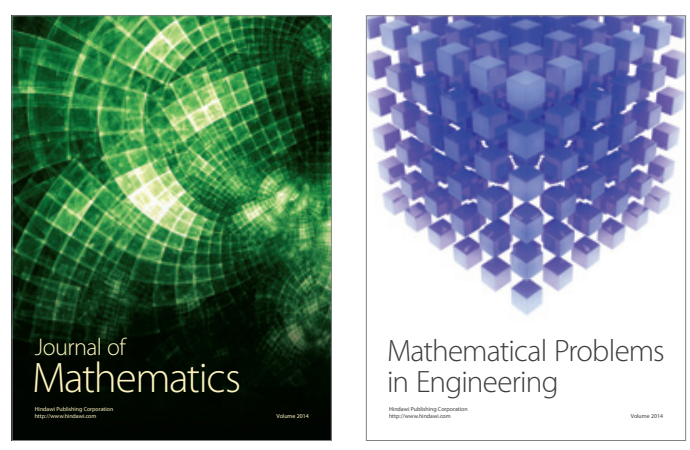

Mathematical Problems in Engineering
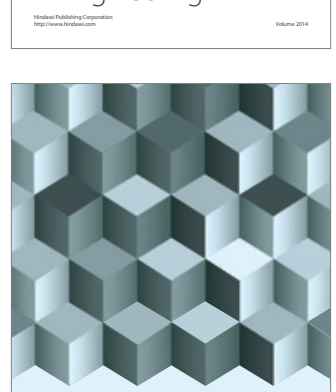

Journal of

Function Spaces
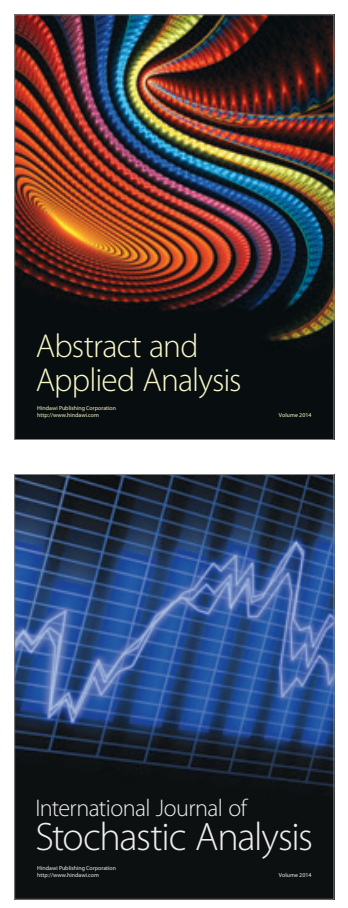

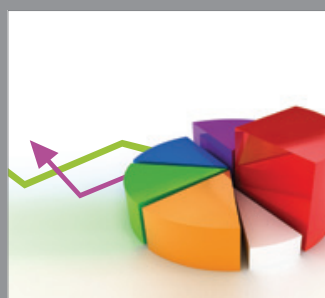

ournal of

Probability and Statistics

Promensencen
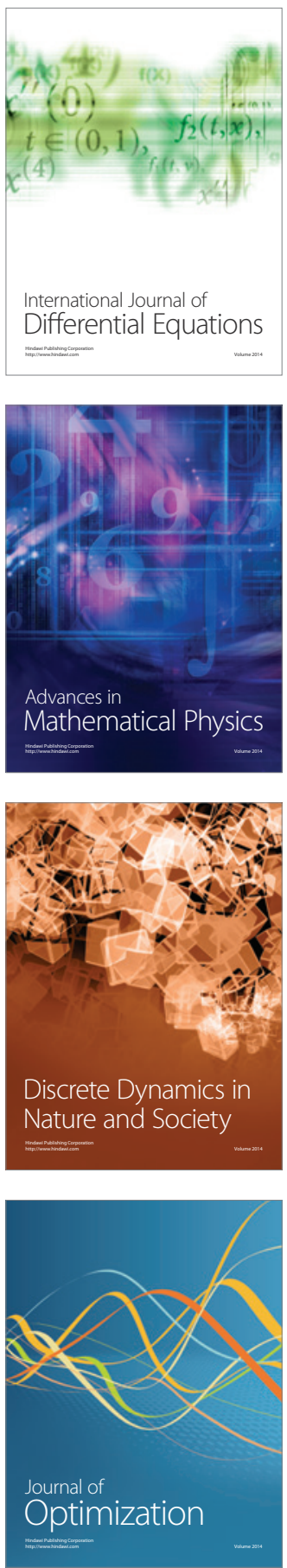